\title{
A survey on the experience of 136 Italian urologists in the treatment of erectile dysfunction with PDE5 inhibitors and recommendations for the use of Avanafil in the clinical practice
}

\author{
Vincenzo Mirone ${ }^{1}$, Ferdinando Fusco ${ }^{1}$, Fabio Parazzini ${ }^{2}$, Alessandro Zucchi ${ }^{3}$ \\ ${ }^{1}$ Dipartimento Ostetricia, Ginecologia, Urologia - Clinica Urologica Università di Napoli Federico II, Italy; \\ 2 I Clinica Ginecologica, Fondazione IRCCS Ca' Granda, Ospedale Maggiore Policlinico, Università di Milano, Italy; \\ ${ }^{3}$ Clinica Urologica ed Andrologica, Università di Perugia, Italy.
}

\begin{abstract}
Summary Introduction: PDE5 inhibitors are the firstline treatment for erectile dysfunction. Although all these drugs share the same mechanism of action, each agent could have different characteristics in terms of selectivity, pharmacokinetics and tolerability profile. Materials and Methods: This manuscript illustrates a project, undertaken by the Italian Society of Urology in order to obtain a "snapshot" of the experience of Italian urologists with the use of PDE5 inhibitors in the clinical practice. This project included a survey, targeting a sample of 136 Italian urologists experienced in the treatment of ED, and the organization of a conference of experts who, based on the findings of the survey, the scientific literature and the clinical experience, would define some recommendations for the use of PDE5 inhibitors in clinical practice with a particular focus on Avanafil, the most recent drug in this class.

Results: The following recommendations on the use of Avanafil were issued: 1) In patients who are candidates for the use of Avanafil, it is advisable to use the 200-mg dose from the first administration; 2) When used at the highest dose (200 mg), Avanafil shows a favourable tolerability profile with an efficacy similar to that of other agents;

3) The patient should be instructed to take Avanafil on an empty stomach, i.e., 30-45 minutes before or 2 hours after a meal; 4) The efficacy window of Avanafil is between 30 minutes and 6 hours after dosing, which qualifies this molecule as a new drug with an intermediate duration of action;

5) Avanafil at a dose of 50-100 mg/day may be a therapeutic option in chronic rehabilitation.

Conclusions: Among PDE5 inhibitors, Avanafil is a new agent with an intermediate duration of action, characterized by high efficacy and good tolerability even at the highest dose (200 mg).
\end{abstract}

KEY WORDS: Erectile dysfunction; PDE5 inhibitors; Avanafil; Italian survey; Recommendations; Dosage.

Submitted 1 June 2016; Accepted 10 June 2016

\section{INTRODUCTION}

Erectile dysfunction (ED), defined as the consistent or recurrent inability to attain and maintain an erection sufficient for satisfactory sexual performance, is a condi- tion with a high prevalence worldwide; it has been estimated that $5-20 \%$ of men are affected by moderate-tosevere ED at some time during their sexual life (1).

The introduction in the clinical practice of phosphodiesterase type 5 inhibitors (PDE5is), in the early 1990s, represented a milestone in the treatment of ED.

Penile erection is a complex neurovascular event that occurs due to relaxation of cavernosal helicine arteries and smooth muscle of corpora cavernosa in combination with a coincident veno-occlusion, leading to blood distension of the corporal sinusoid and resultant penile rigidity (2). The primary mediator of this process is the nitric oxide (NO)/cyclic guanosin-monophosphate (cGMP) pathway. Phosphodiesterase type 5 (PDE5), the enzyme which breakdowns cGMP in the corpus cavernosum, regulates the NO-mediated relaxation of smooth muscle cells. The mechanism of action of PDE5is consists precisely in the inhibition of PDE5, which results in increased cGMP concentrations with continued activation of the NO/cGMP pathway and subsequent increase in blood flow into the corpora cavernosa (3).

The efficacy of PDE5 inhibitors is independent of patient age, etiology of ED (organic, psychogenic, mixed), and baseline severity of the condition (4).

Four PDE5is (Sildenafil, Tadalafil, Vardenafil, and Avanafil) have so far been approved for clinical use in Italy.

Avanafil, the most recently marketed molecule, differs pharmacologically from the other agents of the same class due to its higher selectivity for the PDE5 isoenzyme. Indeed, PDE5 inhibitors have different selectivities for this isoenzyme, as they may exert inhibitory effects on other PDE isoforms resulting in potential side effects, sometimes serious enough to require treatment discontinuation. On the other end, the greater the selectivity for the PDE5 isoenzyme, the less likely is the occurrence of adverse events potentially related to the inhibition of other PDE isoforms (5). Experimental studies have shown that Avanafil has higher selectivity for PDE5 than for other isoenzymes. It is generally believed that the high selectivity of Avanafil results in better tolerability compared with other less selective agents $(5,6)$. 
The efficacy of Avanafil in the treatment of ED is confirmed by a number of double-blind, placebo-controlled, randomized clinical trials, conducted both in the general population (7) and in "difficult" patient subgroups, such as those with diabetes mellitus (8) or those who have undergone nerve-sparing radical prostatectomy (9). In these clinical trials, Avanafil demonstrated a rapid onset of action, often as early as 15 minutes after dosing, a prolonged therapeutic effect (in some cases up to 6 hours after administration), and a good tolerability (10). After approximately two years from the Avanafil launch in Italy, urologists have now developed a specific clinical experience with the use of this drug. However, there are still some "uncertainties" about the best use of Avanafil, particularly with regard to dosage, efficacy, and safety profile compared with older PDE5is.

In order to obtain a "snapshot" of the use of Avanafil in the clinical practice, and to make recommendations for its "best" use, the Italian Society of Urology (SIU, Società Italiana di Urologia) started a project involving:

1) a survey, targeting a sample of Italian urologists experienced in the treatment of ED, with a particular focus on their clinical experience with the use of Avanafil; and

2) the organization of a conference of experts who, based on the findings of the survey and the scientific literature, would define some recommendations for the use of Avanafil in clinical practice.

This paper describes, in its first part, the preliminary results of the survey, and subsequently presents the recommendations made by the conference of experts.

\section{MATERIAL AND METHODS}

This research was conducted from February to April 2016 through an online survey. SIU members were invited to answer anonymously a number of questions included in a questionnaire. In particular, SIU local chairpersons and physicians experienced in the treatment of ED were invited to participate.

The questionnaire investigated the number of ED patients treated over the past 12 months, and the proportion of them treated with Avanafil. Physicians were also asked to indicate, based on their clinical experience, the characteristics of the patients (expressed in percentage) who had been treated with Avanafil $100 \mathrm{mg}$ and $200 \mathrm{mg}$. The aim of this survey was to collect information on physicians' experience in this context; therefore, detailed information on individual patients was not collected.

A total of 136 urologists responded to the survey (88.2\% male; mean age 47 years, range $26-71$ years; $48.5 \%$ of the respondents stated they worked mainly in a hospital setting).

Overall, urologists who participated in this survey stated that they had treated 17.856 patients during the 12 months prior to the completion of the questionnaire. Of these, $13 \%$ were treated with Avanafil $100 \mathrm{mg}$, and $26 \%$ with Avanafil $200 \mathrm{mg}$ (the same patient could have been treated, at different times, with both doses of Avanafil).

Table 1 shows the characteristics of the patients treated with Avanafil $100 \mathrm{mg}$ and $200 \mathrm{mg}$, the patient satisfaction, and the frequency of side effects.

Patients treated with Avanafil 200 mg were on average older and were more likely to report comorbid diabetes mellitus. The use of Avanafil $100 \mathrm{mg}$ was more common in treatment-naive patients. Patient satisfaction was greater in those treated with Avanafil $200 \mathrm{mg}$. Likewise, the frequency of side effects was slightly higher in individuals treated with the higher dose of Avanafil: overall, the observed difference in the frequency of side effects was essentially due to a higher frequency of headaches.

The methodology chosen for the production of recommendations was that of the "consensus conference", which involves the drafting of recommendations by a "jury" at the end of a presentation and consultation of experts summa-

\section{Table 1.}

Characteristics of patients receiving Avanafil $100 \mathrm{mg}$ and $200 \mathrm{mg}$ as reported by physicians participating in the SIU survey.

\begin{tabular}{|lcc|}
\hline Patient characteristics & Patients treated with Avanafil $\mathbf{1 0 0 ~} \mathbf{~ m g ~ ( \% )}$ & Patients treated with Avanafil 200 $\mathbf{~ m g ~ ( \% ) ~}$ \\
\hline Age (years) > 50/<50 & $61.6 / 38.4$ & $65.7 / 34.3$ \\
\hline Organic-based ED & 56.6 & 51.1 \\
\hline Comorbid diabetes mellitus & 28.6 & 36.2 \\
\hline First treatment (treatment-naïe pts) & 45.7 & 40.4 \\
\hline On-demand use & 65.4 & 63 \\
\hline Patient satisfaction (yes/no) & $54.2 / 45.8$ & $58.4 / 41.6$ \\
\hline Frequency of side effects & & 22.4 \\
Any side effects & 15.9 & 16.5 \\
Headache* & 12.6 & 4.1 \\
Hot flushes & 5.5 & 1.1 \\
Back ache & 2.5 & 2 \\
Rash/itching & 2.6 & \\
\hline * Only the side effects reported in more than 2\% of the patients in at least one of the two groups are listed. \\
The percentages shown are mean percentages, weighted by the number of patients treated by each physician. & \\
\hline
\end{tabular}


rizing scientific knowledge on a given topic. The critical analysis of the literature enables the "jury" to compare the available evidence with expert opinions or reports.

This method implies the following steps:

1. the definition of the themes of the recommendations;

2. the search for relevant literature;

3. the preparation of the first draft of the consensus statement by a drafting group;

4. the discussion by a "jury" of experts, and the preparation of the final document.

In March 2016, the drafting group identified the themes of the recommendations, searched PubMed using the key words "avanafil, erectile dysfunction, treatment", and then prepared a first draft of the recommendations.

The experts invited to take part in the production of the recommendations participated in a workshop that was held on April 21-22, 2016. During this workshop, the results of the literature review and the findings of the SIU survey, reported in the introduction of this paper, were presented.

Subsequently, the recommendations prepared by the drafting group were also presented.

The expert panel was composed of 16 SIU local chairpersons and 32 experts ( 2 per local area) in the treatment of ED, identified among the SIU members by the local chairpersons of the association.

\section{RESULTS}

All the experts discussed, amended, and approved in plenary session the recommendations that are presented herein, with comments illustrating their rationale and relevance for the clinical practice.

1. In patients who are candidates for the use of Avanafil, it is advisable to use the 200-mg dose from the first administration.

The data of this survey indicate that the 200-mg dose of Avanafil is the most commonly used in clinical practice. This practice is supported at least partly by scientific literature. Corona et al. (11) have recently published a systematic review of the literature on randomized controlled trials (RCTs), evaluating Avanafil versus placebo in the treatment of erectile dysfunction. This systematic review included five placebo-controlled RCTs, showing that Avanafil was superior to placebo in improving vaginal penetration and achieving a successful sexual intercourse. This review of the literature concluded that both the 100- and 200-mg doses of Avanafil were effective and well tolerated. Actually, the single most commonly reported adverse effect with Avanafil $200 \mathrm{mg}$ was an increased frequency of headache compared to Avanafil $100 \mathrm{mg}$, although this difference was not statistically significant (this finding has also been reported in the present survey on the clinical practice in Italy). No differences were observed between the two groups in the rate of treatment continuation.

With regard to efficacy, the analysis of published studies indicates a greater efficacy (although not statistically significant) of the treatment with the 200-mg dose. The effect of Avanafil $100 \mathrm{mg}$ was also lower in elderly subjects, while no differences were observed with Avanafil $200 \mathrm{mg}$.

The observation that the 200-mg dose of Avanafil shows greater efficacy in all patient categories, while having a substantially similar tolerability profile, clearly supports the recommendations of our working group.

2. When used at the highest dose (200 mg), Avanafil shows a favourable tolerability profile with an efficacy similar to that of other agents.

Chen et al. (12) have recently published a "network metaanalysis" that included RCTs evaluating the efficacy of Sildenafil, Tadalafil, Vardenafil, and Avanafil, usually in comparison with placebo. Overall, the authors included 82 RCTs (for a total of 47626 patients) for the efficacy analysis, and 72 RCTs (20325 patients) for the tolerability analysis.

The authors concluded that there were no significant differences between Avanafil, Sildenafil, Tadalafil, and Vardenafil, when the maximum recommended doses of these drugs (200, 100, 20 and $20 \mathrm{mg}$, respectively) were administered. With regard to tolerability, Avanafil was significantly associated with a lower incidence of adverse effects when drugs were given at the maximum recommended dosage patients were treated with the maximum recommended doses of these drugs $(200,100,20$, and 20 $\mathrm{mg}$, respectively).

\section{The patient should be instructed to take Avanafil on} an empty stomach, i.e., 30-45 minutes before or 2 hours after a meal.

It is important to adequately educate the patient about the fact that Avanafil should be taken on an empty stomach. In fact, the median Time to Maximum Plasma Concentration $\left(\mathrm{T}_{\max }\right)$ is obtained within 30-45 minutes after dosing, and the concomitant intake with food causes a delay in median $T_{\max }$ of 1 hour and 25 minutes, as reported in the Summary of Product Characteristics (SPC) of Avanafil (13).

\section{The efficacy window of Avanafil is between 30 minutes and 6 hours after dosing, which qualifies this molecule as a new drug with an intermediate duration of action.}

Table 2 shows the median Tmax and the plasma half-life (in hours) of the four PDE5 inhibitors available today in Italy for the treatment of ED (13-17). With regard to Avanafil, discordant data about half-life have been presented in the literature. Its terminal half-life is 6-17 h, as reported in the SPC, while the apparent T1/2 is 1.20 to
Table 2.

$T_{\max }$ and plasmatic half-life of PDE5-inhibitors (13-17).

\begin{tabular}{|lcccc|}
\hline Parameter & Avanafil & Sildenafil & Vardenafil & Tadalafil \\
\hline $\mathrm{T}_{\max }($ median) & $30-45 \mathrm{~min}$ & $1 \mathrm{~h}$ & $1 \mathrm{~h}$ & $2 \mathrm{~h}$ \\
\hline Plasmatic half-life & $1.20-5 \mathrm{~h}$ & $3-5 \mathrm{~h}$ & $4-5 \mathrm{~h}$ & $17.5 \mathrm{~h}$ (mean) \\
\hline
\end{tabular}


5 hours (17), in line with the duration of the therapeutic effect observed in clinical trials.

It is quite clear from above that Avanafil can be qualified as a new agent with an intermediate duration of action, as compared with other active substances.

\section{Avanafil at a dose of 50-100 mg/day may be a thera- peutic option in chronic rehabilitation.}

There is scientific evidence supporting the use of PDE5 inhibitors as on-demand or chronic (daily) treatment for penile rehabilitation following radical prostatectomy. The fibrosis of cavernous bodies has been associated with reduced penile length: experimental studies have demonstrated that, following bilateral cavernous nerve injury, there is a significant reduction in the cavernosal smooth muscle/collagen ratio. Chronic use of PDE5is has been specifically studied with the aim to improve this clinical problem. Avanafil has been shown to be effective in the treatment of patients with ED following radical prostatectomy. When prescribed for chronic use, these agents are usually given at the lowest dose available (18), and increasing their dose does not seem to improve their efficacy (19).

\section{Discussion}

As emphasized by the recent guidelines of the European Association of Urology (EAU), PDE5is are the first-line therapeutic option for most men with ED. Currently available PDE5is (Sildenafil, Tadalafil, Vardenafil, and Avanafil) have the same mechanism of action; therefore, their efficacy is substantially similar at comparable doses. There are, however, relevant differences in the pharmacokinetic profile and degree of selectivity between different agents, and this should be taken in account in the therapeutic decision-making process.

The high selectivity of Avanafil, in particular, gives this agent a very satisfactory tolerability profile, as pointed out also by one of the recommendations in this paper, which suggests that good tolerability is one of the main "strengths" of Avanafil compared to other molecules.

The prevailing opinion among the participants in this survey is that, in patients who are candidates for the use of Avanafil, the 200-mg dose should be used from the start of the treatment; in fact, this dose provides an appropriate balance between the efficacy and tolerability of PDE5is, in combination with satisfactory response and compliance rates. According to the findings of this survey, there seem to be no significant differences in terms of efficacy between Avanafil, Sildenafil, Tadalafil, and Vardenafil when the maximum recommended doses of these drugs are used $(200,100,20$, and $20 \mathrm{mg}$, respectively); moreover, Avanafil shows a favourable tolerability profile when used at the highest dose $(200 \mathrm{mg})$, with an efficacy similar to that of other agents.

A crucial point for the successful treatment of ED with PDE5is is the effectiveness of Doctor-Patient communication in relation to the proper administration of prescribed medications and the outcomes to be expected, both in terms of efficacy and response times. In fact, if these issues are not adequately explained to the patients, they may mistakenly believe that the treatment pre- scribed is ineffective and/or may inappropriately stop or change their medications on their own initiative.

In particular, the patient should be instructed to take Avanafil on an empty stomach, i.e., 30-45 minutes before or 2 hours after a meal, in order to obtain an optimal therapeutic effect; it is also necessary to explain that the efficacy window of Avanafil is between 30 minutes and 6 hours after dosing, which qualifies this new PDE5i as an agent with intermediate duration of action.

Finally, a topic of great interest that has emerged from this survey is that Avanafil, used at a dose of 50-100 $\mathrm{mg} /$ day, can be a therapeutic option for long-term penile rehabilitation, confirming the literature data that support the use of PDE5-inhibitors as on-demand or chronic treatment for penile rehabilitation after radical prostatectomy (20).

\section{Conclusions}

Avanafil, a highly selective PDE5 inhibitor, characterized by rapid onset of action and prolonged therapeutic effect, is an effective and well tolerated option for the treatment of ED. The present survey, conducted by SIU among Italian urologists, largely confirms the clinical efficacy and tolerability of Avanafil in a real-life setting, even at the highest dose $(200 \mathrm{mg})$, in line with the results of previous international clinical trials conducted to evaluate this PDE5 inhibitor.

\section{REFERENCES}

1. Hatzimouratidis K, Eardley I, Giuliano F, et al. Guidelines on male sexual dysfunction: erectile dysfunction and premature ejaculation. European Association of Urology Web site. http://uroweb.org/guideline/male-sexual-dysfunction/. Updated 2015.

2. Ali W, Besarani D, Kirby R. Modern treatment of erectile dysfunction. Br J Diabetes Vasc Dis. 2002; 2:255.

3. Limin M, Johnsen N, Hellstrom WJ. Avanafil, a new rapid-onset phosphodiesterase 5 inhibitor for the treatment of erectile dysfunction. Expert Opin Investig Drugs. 2010; 19:1427.

4. Bruzziches R, Francomano D, Gareri P, et al. An update on pharmacological treatment of erectile dysfunction with phosphodiesterase type 5 inhibitors. Expert Opin Pharmacother. 2013; 14:1333.

5. Gupta R, Kumar G, Kumar RS. An update on cyclic nucleotide phosphodiesterase (PDE) inhibitors: phosphodiesterases and drug selectivity. Methods Find Exp Clin Pharmacol. 2005; 27:101.

6. Omori K, Kotera J. Overview of PDEs and their regulation. Circ Res. 2007; 100:309.

7. Goldstein I, McCullough AR, Jones LA, et al. A randomized, double-blind, placebo-controlled evaluation of the safety and efficacy of avanafil in subjects with erectile dysfunction. J Sex Med. 2012; 9:1122.

8. Goldstein I, Jones LA, Belkoff LH, et al. Avanafil for the Treatment of Erectile Dysfunction: A Multicenter, Randomized, Double-Blind Study in Men With Diabetes Mellitus. Mayo Clin Proc. 2012; 87:843

9. Mulhall JP, Burnett AL, Wang R, et al. A Phase 3, placebo controlled study of the safety and efficacy of avanafil for the treatment of erectile dysfunction after nerve sparing radical prostatectomy. $J$ Urol. 2012; 189:2229. 
10. Belkoff LH, McCullough A, Goldstein I, et al. An open-label, longterm evaluation of the safety, efficacy and tolerability of avanafil in male patients with mild to severe erectile dysfunction. Int J Clin Pract. 2013; 67:333.

11. Corona G, Rastrelli G, Burri A, et al. The safety and efficacy of Avanafil, a new 2(nd) generation PDE5i: comprehensive review and meta-analysis. Expert Opin Drug Saf. 2016; 15:237.

12. Chen L, Staubli SE, Schneider MP, et al. Phosphodiesterase 5 inhibitors for the treatment of erectile dysfunction: a trade-off network meta-analysis. Eur Urol. 2015; 68:674.

13. Avanafil Summary of Product Characteristics (SPC).

14. Sildenafil Summary of Product Characteristics (SPC).

15. Vardenafil Summary of Product Characteristics (SPC).
16. Tadalafil Summary of Product Characteristics (SPC).

17. Kedia GT, Ückert S, Assadi-Pour F, et al. Avanafil for the treatment of erectile dysfunction: initial data and clinical key properties. Ther Adv Urol. 2013; 5:35.

18. Bannowsky A, van Ahlen H, Loch T. Increasing the dose of vardenafil on a daily basis does not improve erectile function after unilateral nerve-sparing radical prostatectomy. J Sex Med. 2012; 9:1448.

19. Bannowsky A, Schulze H, van der Horst C, et al. Recovery of erectile function after nerve-sparing radical prostatectomy: improvement with nightly low-dose sildenafil. BJU Int. 2008; 101:1279.

20. Cui YS, Li N, Zong HT, et al. Avanafil for male erectile dysfunction: a systematic review and meta-analysis. Asian Journal of Andrology. 2014; 16:472.

\section{Correspondence}

Vincenzo Mirone, MD

mirone@unina.it

Ferdinando Fusco, MD (Corresponding Author)

ferdinando-fusco@libero.it

Dipartimento Ostetricia, Ginecologia, Urologia

Clinica Urologica Università di Napoli Federico II, Italy

Fabio Parazzini, MD

fabio.parazzini@unimi.it

I Clinica Ginecologica, Fondazione IRCCS Ca' Granda Ospedale Maggiore Policlinico,

Università di Milano, Italy

Alessandro Zucchi, MD

zucchi.urologia@gmail.com

Clinica Urologica ed Andrologica, Università di Perugia, Italy 